\title{
Qualitative Inquiry into the Patients' Expectations regarding Nurses and Nursing Care
}

\author{
Majid Najafi Kalyani, ${ }^{1}$ Raziyeh Illon Kashkooli, ${ }^{2}$ Zahra Molazem, $^{2}$ and Nahid Jamshidi ${ }^{2}$ \\ ${ }^{1}$ School of Nursing, Fasa University of Medical Sciences, Fasa 7461686688, Iran \\ ${ }^{2}$ Faculty of Nursing and Midwifery, Shiraz University of Medical Sciences, Shiraz 7193613119, Iran \\ Correspondence should be addressed to Majid Najafi Kalyani; majidnajafi5@yahoo.com
}

Received 2 January 2014; Revised 6 March 2014; Accepted 12 March 2014; Published 3 April 2014

Academic Editor: Jenny M. Wilkinson

Copyright (c) 2014 Majid Najafi Kalyani et al. This is an open access article distributed under the Creative Commons Attribution License, which permits unrestricted use, distribution, and reproduction in any medium, provided the original work is properly cited.

Background and Objectives. Awareness about the patients' needs and expectations is quite important in improving the quality of the services they are provided with. Since meeting the needs and expectations of the patients is one of the basic issues in patient care, the present study aimed to investigate the patients' expectations from nurses and nursing care. Methods. In this qualitative study, 20 hospitalized patients were interviewed. The data were gathered through deep, semistructured interviews. Then, all the recorded interviews were transcribed, reviewed for several times, and analyzed using qualitative content analysis approach. Results. By analyzing of data, three main categories were extracted. The main categories of the patients' expectations from nurses and nursing care were comprehensive care, ethical performance, and having proper individual characteristics. Discussion and Conclusion. The study results revealed that the patients expected comprehensive care from the nurses. In addition, the nurses were required to apply the ethical principles in what they do as their duties. The findings of the study can be helpful in improving the patient care.

\section{Introduction}

Nurses, as one of the main and influential members of the healthcare teams, play a critical role in patient care [1]. Moreover, awareness about the patients' concerns, needs, and expectations is of utmost importance for the researchers and providers of nursing care [2]. During the past decades, health researchers have noticed the patients' concerns about their disease and medical care [3]. Thus, understanding and meeting the patients' expectations are one of the major objectives of nursing profession for achieving efficient care for the patients [4].

Patients' expectation from the nurses and other members of the healthcare team refers to their concept of care provision before hospitalization. Of course, patients' expectation does not imply the patients' satisfaction with the provided services. Satisfaction can only be measured during the patients' hospital stay and after being discharged; at the end, the patients may express a high level of satisfaction while their expectations have not been completely met [5]. Thus, investigating and meeting the patients' expectations from care providers are far beyond providing their satisfaction [6]. Most of the patients are aware of their expectations from the nurses [7] and it is necessary to assess their expectations separately from their satisfaction from nursing care $[8,9]$.

Taking the patients' expectations into account also results in the patients playing a more important role as one of the members of the treatment team in improving their own health $[4,10]$. Furthermore, it helps the nurses to decrease the distance between the patients' expectations and their real experiences [11]. Overall, the patients' expectations from nurses and nursing care are affected by their culture, beliefs, attitude, and amount of knowledge [6, 11]. Moreover, nursing care in the intensive care units refers to reassuring the patients who expect to be aware of the nursing care provided for them [10].

Nursing in Iran was begun with the first nursing school that was established in Tabriz in 1916. After the Islamic revolution in 1979, more growth emerged in nursing. There are now more than one hundred nursing schools and three levels of B.S., M.S., and Ph.D. in Iran. Nurses in Iran are working in hospitals and academic centers in the areas of management, treatment, research, and education [12]. 
According to Iran's religious and cultural contexts, these factors may affect the patients' expectations regarding nurses and nursing. Furthermore, the patient expectations can vary based on the cultural and social context of each country [6]; identifying these needs in our country will help nurses and other healthcare members to pay attention to these cases in planning care.

A limited study has been done qualitatively on the expectations of patients regarding nursing in Iran. One study by Kalyani et al. has examined the patients expectations related to coronary angiography [6].

With regard the gap in previous studies and also need to exploring this issue in the sociocultural context of Iran, the current qualitative study aims to investigate the Iranian patients' expectations from nurses and nursing care; the results can be used in order to improve the quality of nursing care and education in Iran healthcare setting.

\section{Methods}

This qualitative study aimed to explore the hospitalized patients' expectations regarding nurses and nursing care. A descriptive exploratory qualitative approach was chosen. This approach was conducted to reach a deep understanding of the patients' expectations regarding nurses and nursing care. The patients were selected through purposeful sampling which is appropriate for qualitative studies. The sampling of patients similar to other qualitative studies continued until the researcher reached saturation. After the interview with 20 patients, the researchers reached saturation.

The study participants were selected from the patients between 49 and 63 years old who had been hospitalized in the coronary care unit (CCU) of one of Shiraz hospitals for at least 3 days. After selecting the patients, the study data were collected through deep, semistructured interviews starting with a general question on the patients' expectations from the nurses and nursing care and, according to the patients' responses, the following questions were selected and asked. It should be noted that the patients were interviewed in the normal conditions of hospitalization and when they were in good general health status. The interviews, which lasted for 30-45 minutes, were recorded using a voice recorder and transcribed verbatim at the end of the interview.

Transcribed data were analyzed using conventional qualitative content analysis. In this way, each transcribed interview was read for several times and the primary codes were extracted. Then, the related codes were put in one group and each code was matched with what the participants had said. Finally, based on similarity and content, the subcategories were used to make the main categories.

To ensure trustworthiness of data, continuous investigation of the data (transcription of the data and investigating them until the main themes were obtained), peer check, and member check were performed. Moreover, the objectivity of the data was determined through continuous, accurate, and proper treatment of all stages of the research study and clarity of the research method. Also, using a team approach in data analysis (i.e., reviewing the data analysis by the research team for increasing the accuracy), the reliability of the results was confirmed.
Prior to the study, the aim of the research and method were explained to the patients, written informed consents were obtained from them, and they were asked for permission for recording their voice. Moreover, the patients were ascertained that the study results would only be used in order to improve the quality of nursing care and the principle of anonymity was observed, as well.

\section{Results}

In this study, $80 \%$ of the participants were male and $20 \%$ female and their mean age was 51 years. After data analysis, 274 primary codes, including 3 main categories and 10 subcategories, were extracted. The main categories of patients' expectations were comprehensive care, ethical performance, and having proper individual characteristics.

3.1. Comprehensive Care. This main category included three subcategories of patient education, physical care, and psychological care.

3.1.1. Patient Training. The study findings showed that most of the patients considered the education provided by nurses as one of their most important expectations. This consisted of education about the disease, explaining the disease-related issues to the patients, and responding to their questions about the disease as well as the medications. For instance, one of the study participants said: "During the two minutes the nurse comes to me and gives me medicine; s/he can tell me what the medicine's name is or why s/he is giving me this medication. I do not know the name of my drugs at all" (Patient 1).

Another patient stated: "Most of the patients come here to be cured. Everything you say is like a verse for them. S/he comes to me and measures my body temperature but I see him/her as a doctor. I tell myself that s/he knows everything and I like him/her to tell me something about my disease" (Patient 7).

One other study participant mentioned: "It is important that the nurse explains our disease for us in 5 minutes. A lot of patients are hospitalized again because their treatment has been ambiguous; they go home and do not know what to do and how to take care of themselves. So, they become ill again and go back to the hospital" (Patient 12).

3.1.2. Physical Care. This subcategory included appropriate, comprehensive care, meeting the patients' needs, sedating the patients' pain, following up the patients, frequent presence by the patients and asking how they feel, immediate response to their needs, paying attention to the patients' individual needs, and providing the patients' comfort.

In this regard, one of the study participants said: "When I have chest pain at midnight, I tell the nurse and s/he comes to me with a sublingual tablet and heals my pain. This is too much for me... rather than when s/he says wait, I will come in a few minutes" (Patient 10).

One other participant of the study believed: "You should ask. You know that this patient has once had a headache. So, you should ask whether s/he is fine or not. It is important for him/her that you ask about his/her pain" (Patient 18). 
3.1.3. Psychological Care. Another subcategory obtained from what the participants had stated was psychological care for the patients. Different dimensions of this subcategory included convincing the patients, calmly speaking with them, reassuring them, not causing them stress, gaining their trust, creating relationships with them, listening to them, and being kind to them.

One of the study participants said: "Here, the patient may become angry and shout. So, the nurse must calmly and kindly speak to the patient and make him/her ashamed. For example, one of the patients had got angry last night, had removed all the serums, and had separated the monitor, but the nurses managed to convince him and bring him back to the bed" (Patient 17).

Moreover, one of the patients stated: "There may be a patient in this department for whom it is very important that somebody is listening to him/her and s/he can easily open his/her heart. Well, this will open a small window of hope for him/her" (Patient 20).

Yet, another patient believed: "The patient cannot build a relationship with the nurse unless s/he trusts him/her and the nurse should start this relationship. You can see that the patients may not be able to start a relationship; so, the nurse must help them and little by little the mutual trust is built" (Patient 11).

Another study participant also said: "Nurses can influence the patients by their words and good behavior. If nurses are kind to a patient, s/he will be their slave. The patients need empathy and this highly affects them, because the patients think that nobody has problems but themselves" (Patient 5).

3.2. Ethical Performance. This main category consisted of two subcategories of responsibility and respecting the patients' rights.

3.2.1. Responsibility. Most of the study subjects expected the nurses to be responsible for the healthcare as well as the patients. Different dimensions of this subcategory included the nurses' feeling of responsibility, not being indifferent, having discipline, and having justice in treating the patients.

For instance, one of the study participants mentioned: "S/he has to treat us in the same manner and cure me whether I am a doctor, engineer, or a worker" (Patient 2).

Another patient said: "Here, the nurse must be responsible; s/he has accepted this responsibility. Every night, I sleep with fear that I may have another stroke and nobody is here" (Patient 9).

Also, one of the patients stated: "See! When I am here, the nurses must feel responsible toward my pain, my disease, and even the money I pay. I must feel relaxed that they care for me. These are really expectations and they must accept the responsibility" (Patient 15).

3.2.2. Respecting the Patients' Rights. Another subcategory extracted from the interviews with the patients was respecting the patients' rights by the nurses. This subcategory included various dimensions, such as respecting the patients and respecting the patients' privacy.

In this regard, one of the patients said: "I say I have pain, I cannot sleep and the nurse says did you smoke in the past? S/he should not have talked to me that way in front of other patients and nurses. I was really offended" (Patient 16).

Another participant mentioned: "They should say the patient who is here and we are measuring his/her blood pressure is a human and should be respected. The relationship between the nurses and patients should be in a way that they respect and trust each other" (Patient 11).

Yet, one other participant stated: "Nursing is different from the past. It has special academic courses. A nurse means an academic, scientific person and we expect a nurse to treat us as an academic person" (Patient 4 ).

3.3. Proper Individual Characteristics. This category included two subcategories of appropriate behavior and love for work.

3.3.1. Appropriate Behavior. All the participants of the present study considered having good behavior as a priority for nursing and patient care. For instance, one of the patients said: "The nurse's behavior is very important. If the nurse's behavior is not good, the patient will be hurt and may never say his/her needs again. S/he may not even be able to tolerate the hospital anymore" (Patient 7).

Another patient also stated: "First, the nurse must have an ideal behavior, s/he must be friendly. In this way, the patient feels that s/he can say her/his needs and ask for the nurse's help. If the nurse is bad-tempered, the patient will get hurt" (Patient 19).

Another participant mentioned: "Sometimes good behaviors are not shown in the nurse-patient relationship. These behaviors are very important for the patients and bad behaviors may create a great distance between the nurse and the patient. This may not be quite important for the nurse because s/he is involved with a large number of other tasks, but it is very important for the patient" (Patient 12).

3.3.2. Love for Work. Most of the patients in this study considered love for work and compassion as the keys to success in patient care. One of the interviewees said: "If the nurse loves his/her work and likes the patients, the patients will understand it even if they are rustic and of low literacy. Look at the patients with love. Be kind" (Patient 13).

Also, another patient mentioned: "A nurse must love his/her patients and work; otherwise, s/he cannot. The patient should feel it and understand that s/he is important for the nurse. The patients understand it and this will have a great influence on their recovery" (Patient 9).

One other participant stated: "This nurse really loved her work in such a way that the patients enjoyed her presence and work. I enjoy seeing her doing her tasks with good will" (Patient 20).

\section{Discussion}

The findings of the current study presented a clear image of the patients' expectations from nurses and nursing care. The study participants stated their expectations from nurses and nursing care through three main categories of comprehensive 
care, responsibility, and having proper individual characteristics.

All the patients taking part in the present study mentioned their education by nurses as one of their major expectations and believed that educating the patients regarding their disease, experiments, examinations, disease stages, disease future, and diet comprises a main part of nursing care. In the same line, Noohi and Pouraboli showed that although the patients had a high amount of educational expectations and tendency to gain information from the nurses, their educational needs had not been desirably met, resulting in their dissatisfaction [13]. Goupy et al. also showed that, in both types of care, the lowest level of satisfaction was related to the educational dimensions and providing the patients with the necessary information [14]. Unfortunately, in spite of the great importance of patient education, this issue is rarely planned and performed as a formal process during the patients' hospital stay and is mostly carried out informally during the nursing care and the patients' activities [1, 15]. Nurses should examine the patients and identify their needs and, according to their educational needs, prioritize, plan, perform, and assess the patient training programs based on their level of education, culture, facilities, and duration of hospital stay $[6,13]$.

Another important dimension of the patients' expectations from nurses and nursing care was comprehensive or holistic care and the time spent by nurses for the patients. The patients expected the nurses to comprehensively take care of them, build a friendly relationship with them, listen to them, speak with them calmly, meet their needs, reduce their pain, follow up their pain, be with them frequently, and respond to their needs immediately. Jolaee et al. showed that caring presence of the nurses is one of the major dimensions of actual care from the patients' as well as their companions' point of view [16]. Moreover, Benner believes that the nurses' physical presence by the patients for doing the daily duties is not enough for responding to their needs; the patients' psychological as well as emotional needs should be taken into account, as well [17]. In that study, not caring for the patients did not mean lack of physical presence; on the other hand, it referred to being present just for doing one's duties [16, 17].

Overall, the studies conducted on the issue show that the patients expect the nurses to spend more time for their care $[6,16,18]$. In addition, the patients consider the nurses' empathy and caring presence by their bedside as one of their primary rights [16]. Molazem et al. also stated that the presence of the nurses by the patients' bedside leads to the patients feeling of security and tranquility [18]. Evidence shows that the nurses' presence has a large number of positive effects on the patients, including peace of mind, reduction of fear and stress, confidence, and security $[6,16$, 18, 19]. Furthermore, Tschudin believes that talking to the patients and their family members is not a simple task and nurses usually use the lack of enough time as an excuse in order to avoid it [20]. Nevertheless, spending even a short time in speaking with the patients decreases the negative consequences of lack of relationship, such as the patients' dissatisfaction, anxiety, and anger $[6,16]$.
Moreover, the nurses' behaviors including paying attention to the patients, meeting the patients' needs and communication were the index of their competency from the patients' point of view [21]. From the patients' point of view, paying more attention to their affairs by the nurses induces the feeling that they care more about their duties. In addition, the nurses will be considered as the individuals who have no authority and just carry out the orders of other medical consultants [22]. Jouybari et al. defined calming nurses as those who provided care with technical competency and human behavior [22]. In the present study, nurses' availability, for instance, immediate response to the alarm, was one of the main issues which led to the patients' peace of mind. This finding has been confirmed in a great number of studies, such as those conducted by Molazem et al. [18], Jolaee et al. [16], and Kalyani et al. [6].

Overall, the review of the studies shows that although the patients' conceptions of comfort, care, and good nurse have slight differences in various cultures, they have several common points, as well [22]. In fact, the patients' expectations regarding such features as presence, assurance, giving information, listening, paying attention to the patient, and respect show that, in addition to the technical capabilities, artistic dimensions of nursing are also of great importance from the patients' perspective $[16,22]$.

Another important category of the patients' expectations from nurses and nursing care in this study was responsibility and respecting the patients' rights. The patients expected the nurses to feel responsible toward them and, in addition to performing their treatment and care duties, respect their privacy and rights. Consistently, Jouybari et al. showed that the patients considered the nurses' responsibility as a major dimension of nursing care [22]. From the patients' point of view, a responsible and committed nurse is the one who pays special attention to the patients and provides them with a different type of care from others [23]. The patients taking part in the study by Feizi et al. believed that the nurses must be responsible for their behaviors as well as the outcomes of their work and know who they are caring about [24]. In the study conducted by Hunt, the patients stated that, in cardiac intensive care, they would like an experienced, intelligent, and skillful nurse who considers all their needs and helps them all the time to take care of them [10]. In fact, the most important expectation of these patients was education which was highly effective in reducing their fear, anxiety, and discomfort [10]. Consistent with the findings of the present study, the patients' expectations in the study by Watt et al. included the appropriate relationship of the treatment team with the patients, prioritizing the needs and the necessary care for the patients, performing each care service according to the priorities, providing high quality healthcare, and training the patients [5].

Our findings confirm good behavior and love for work as one of the most important patients' expectations regarding nurses. All the participants in our study confirmed the necessity of good individual characteristics and love for work for nurses. Feizi et al. found the nurses' tendency to help the patients as one of the major characteristics of the nurses and expected them to be interested in taking care of the patients 
[24]. Attree also revealed the nurses' inclination as one of the characteristics of a good caregiver which the patients expected from their care providers [25].

The results of this study will help nurses to take better care of these patients. The results showed that patients require holistic care from nurses as well as respect to their values and religious beliefs.

Our findings revealed many aspects of patient's expectations regarding nurses and nursing care and gave a new window for nurses to care for patients. Since our study reflects the viewpoints of a limited number of patients, we cannot generalize the results to all the patients. Furthermore, as all of the patients in this study were cardiac patients, there is a need to conduct a study on what are the expectations of other patients with different disease and ward.

\section{Conclusion}

We aimed to explore the cardiac patient's expectations regarding nurses and nursing care. It is important for nurses to pay attention to the patients' expectations in order to provide better care based on patient's needs. We found that cardiac patients have diverse expectations from the nurses and nursing care. Nurses, as the main personnel of medical team, should include these expectations in their care program and management of cardiac patients. Paying attention to patient's expectations and needs resulted in patient's satisfaction and decrease in hospital stay. Furthermore, attention to the moral and religious beliefs of patients is an important expectation.

\section{Conflict of Interests}

The authors declare that there is no conflict of interests regarding the publication of this paper.

\section{Acknowledgment}

The authors would like to thank Dr. Nasrin Shokrpour at the Center for Development of Clinical Research of Nemazee Hospital for editorial assistance.

\section{References}

[1] N. Jamshidi, A. Abbaszadeh, M. N. Kalyani, and F. Sharif, "Effectiveness of video information on coronary angiography patients' outcomes," Collegian, vol. 20, no. 3, pp. 153-159, 2013.

[2] R. L. Kravitz, "Measuring patients' expectations and requests," Annals of Internal Medicine, vol. 134, no. 9, pp. 881-888, 2001.

[3] S. C. Smeltzer and B. Bare, Brunner and Suddarth's Text Book of Medical Surgical Nursing, Lippincott Williams \& Wilkins, Philadelphia, Pa, USA, 12th edition, 2010.

[4] N. Junod Perron, F. Secretan, M. Vannotti, A. Pecoud, and B. Favrat, "Patient expectations at a multicultural out-patient clinic in Switzerland," Family Practice, vol. 20, no. 4, pp. 428-433, 2003.

[5] D. Watt, W. Wertzler, and G. Brannan, "Patient expectations of emergency department care: phase I-a focus group study," Canadian Journal of Emergency Medicine, vol. 7, no. 1, pp. 1216, 2005.
[6] M. N. Kalyani, F. Sharif, F. Ahmadi, and M. T. Iman, "Iranian patients' expectation about coronary angiography: a qualitative study," Iranian Journal of Nursing and Midwifery Research, vol. 18, no. 3, pp. 180-185, 2013.

[7] S. A. Redsell, C. Jackson, T. Stroke, A. Hastings, and R. Baker, "Patient expectations of "first-contact care" consultations with nurse and general practitioners in primary care," Quality in Primary Care, vol. 15, no. 1, pp. 5-10, 2007.

[8] K. S. Chunta, "Expectations, anxiety, depression, and physical health status as predictors of recovery in open-heart surgery patients," Journal of Cardiovascular Nursing, vol. 24, no. 6, pp. 454-464, 2009.

[9] C. E. Guerra, V. J. McDonald, K. L. Ravenell, D. A. Asch, and J. A. Shea, "Effect of race on patient expectations regarding their primary care physicians," Family Practice, vol. 25, no. 1, pp. 49$55,2008$.

[10] J. M. Hunt, "The cardiac surgical patient's expectations and experiences of nursing care in the intensive care unit," Australian Critical Care, vol. 12, no. 2, pp. 47-53, 1999.

[11] J. H. Farooqi, "Patient expectations of general practitioner," Middle East Journal of Family Medicine, vol. 3, no. 3, pp. 6-9, 2005.

[12] Z. Farsi, N. Dehghan-Nayeri, R. Negarandeh, and S. Broomand, "Nursing profession in Iran: an overview of opportunities and challenges," Japan Journal of Nursing Science, vol. 7, no. 1, pp. 9-18, 2010.

[13] E. Noohi and B. Pouraboli, "Educational requirements of the discharged patients and their satisfaction about nursing educational performance, Kerman, Iran," Hormozgan Medicine Journal, vol. 13, no. 3, pp. 2006-2012, 2008.

[14] F. Goupy, O. Ruhlmann, O. Paris, and B. Thélot, "Results of a comparative study of in-patient satisfaction in eight hospitals in the Paris region," International Journal for Quality in Health Care, vol. 3, no. 4, pp. 309-315, 1991.

[15] N. Jamshidi, A. Abbaszadeh, and M. N. Kalyani, "Effects of video information on anxiety, stress and depression of patients undergoing coronary angiography," Pakistan Journal of Medical Sciences, vol. 25, no. 6, pp. 901-905, 2009.

[16] S. Jolaee, A. Nikbakht Nasrabadi, and Z. Parsa Yekta, “The view of patients and patients' companions regarding patients'rights: a phenomenological research," Hayat, vol. 10, no. 4, pp. 5-20, 2004.

[17] P. Benner, Integrating Phenomenology: Embodiment, Caring and Ethics in Health and Illness, Sage Publication, Thousand Oaks, Calif, USA, 1994.

[18] Z. Molazem, F. Ahmadi, E. Mohammadi, and S. Bolandparvaz, "Improvement in the nursing care quality in general surgery wards: Iranian nurses' perceptions," Scandinavian Journal of Caring Sciences, vol. 25, no. 2, pp. 350-356, 2011.

[19] Z. Molazem and S. Ghadakpour, "What are the most important emotional needs of patients in Iran?" Archives Des Siences, vol. 66, no. 3, pp. 271-278, 2013.

[20] V. Tschudin, Ethics in Nursing, the Caring Relationship, Butterworth Heinemann, Edinburgh, UK, 3rd edition, 2000.

[21] M. Lotzkar, "An observational study of the development of a nurse-patient relationship," Clinical Nursing Research, vol. 10, no. 3, pp. 275-294, 2001.

[22] L. M. Jouybari, F. Haghdoost Oskouee, and F. Ahmadi, "Comforting nurse: patients' experiences and perceptions," Journal of Iranian Medicine University, vol. 12, no. 45, pp. 59-68, 2006. 
[23] A. Fakhr-Movahedi, M. Salsali, R. Negarandeh, and Z. Rahnavard, "Exploring contextual factors of the nurse-patient relationship: a qualitative study," Koomesh, vol. 13, no. 1, pp. 23-34, 2011.

[24] A. Feizi, R. Mohammadi, and M. Nikravesh, "Factors causing patient's trust in nurse from patients' perspective," Razi Journal of Medical Sciences, vol. 13, no. 52, pp. 177-187, 2006.

[25] M. Attree, "Patients' and relatives' experiences and perspectives of "good" and "not so good" quality care," Journal of Advanced Nursing, vol. 33, no. 4, pp. 456-466, 2001. 


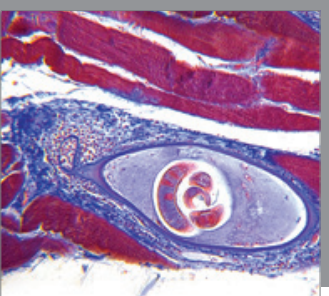

Gastroenterology

Research and Practice
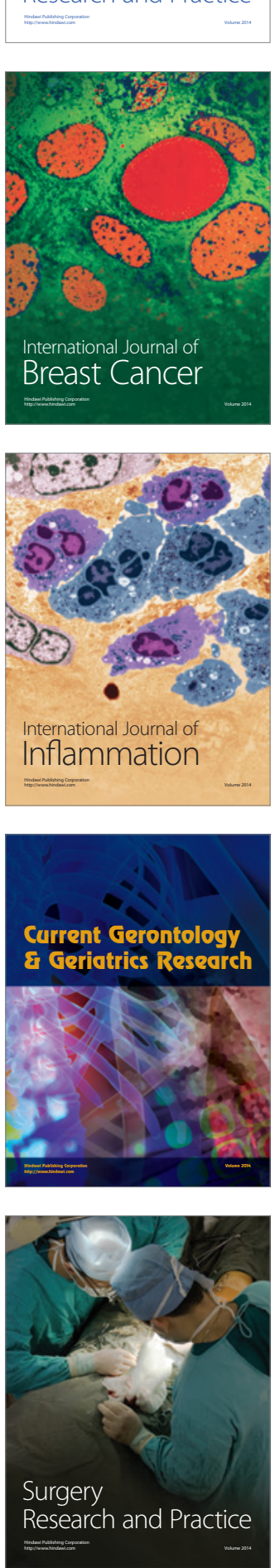

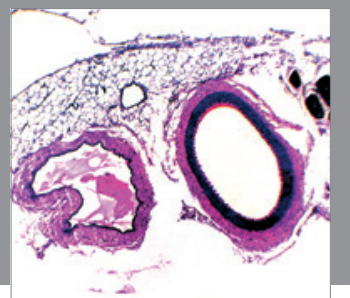

International Journal of Hypertension
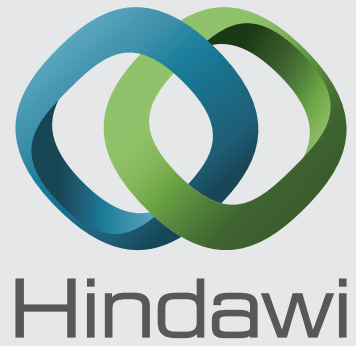

Submit your manuscripts at http://www.hindawi.com
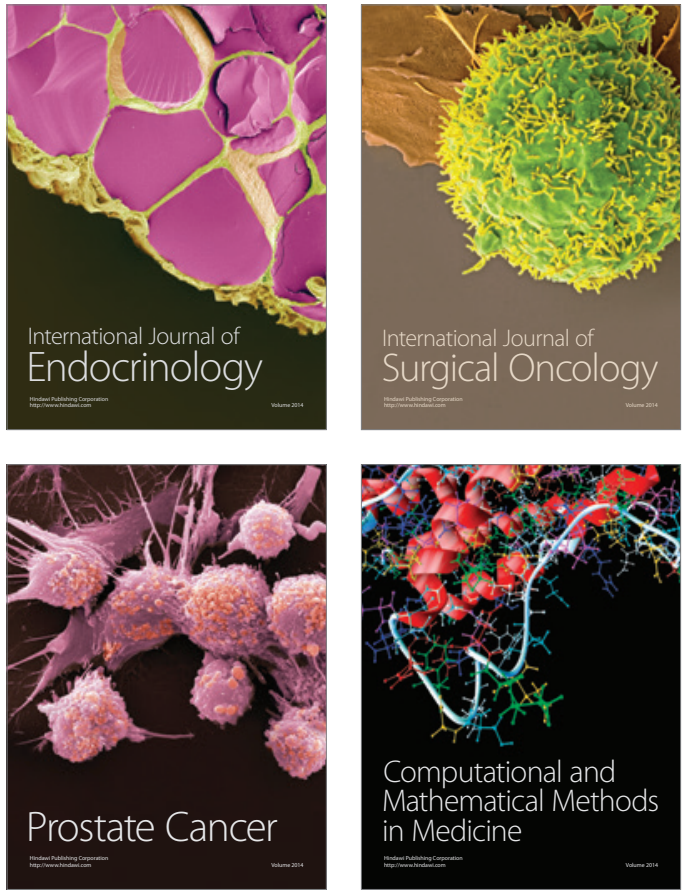
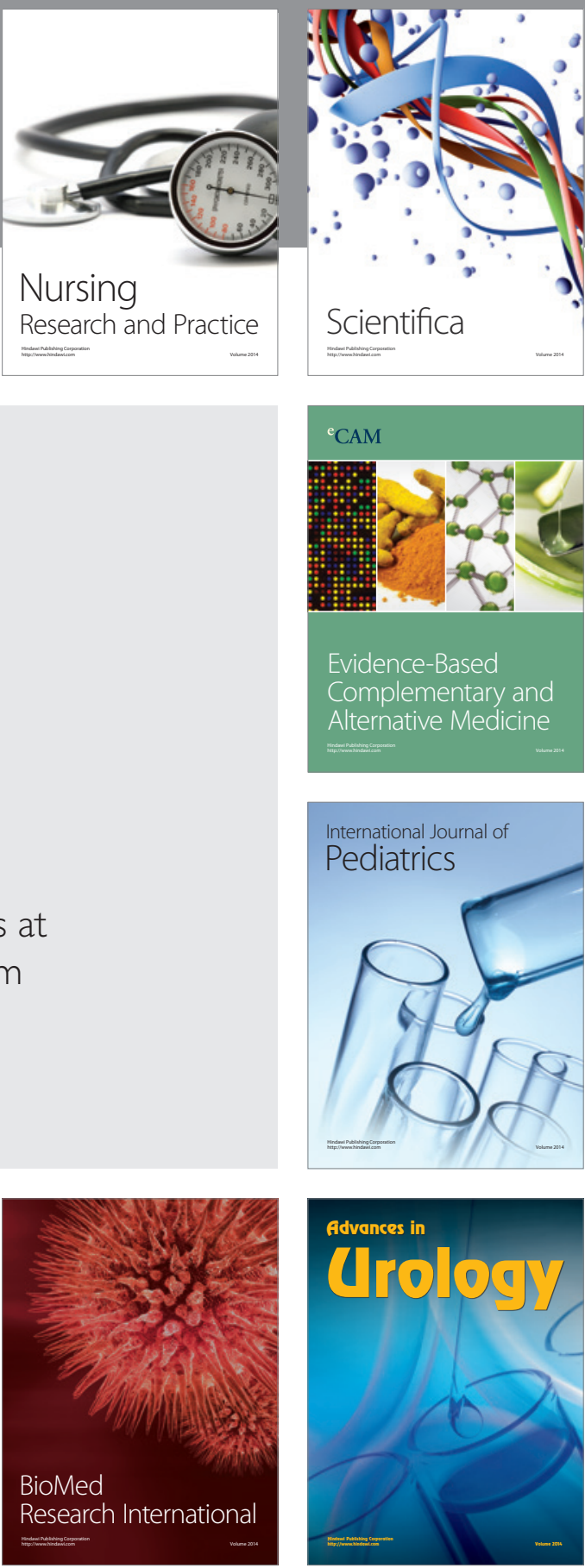

Nursing

Research and Practice

Scientifica

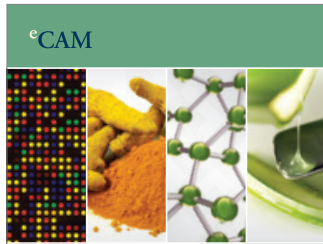

Evidence-Based

Complementary and Alternative Medicine
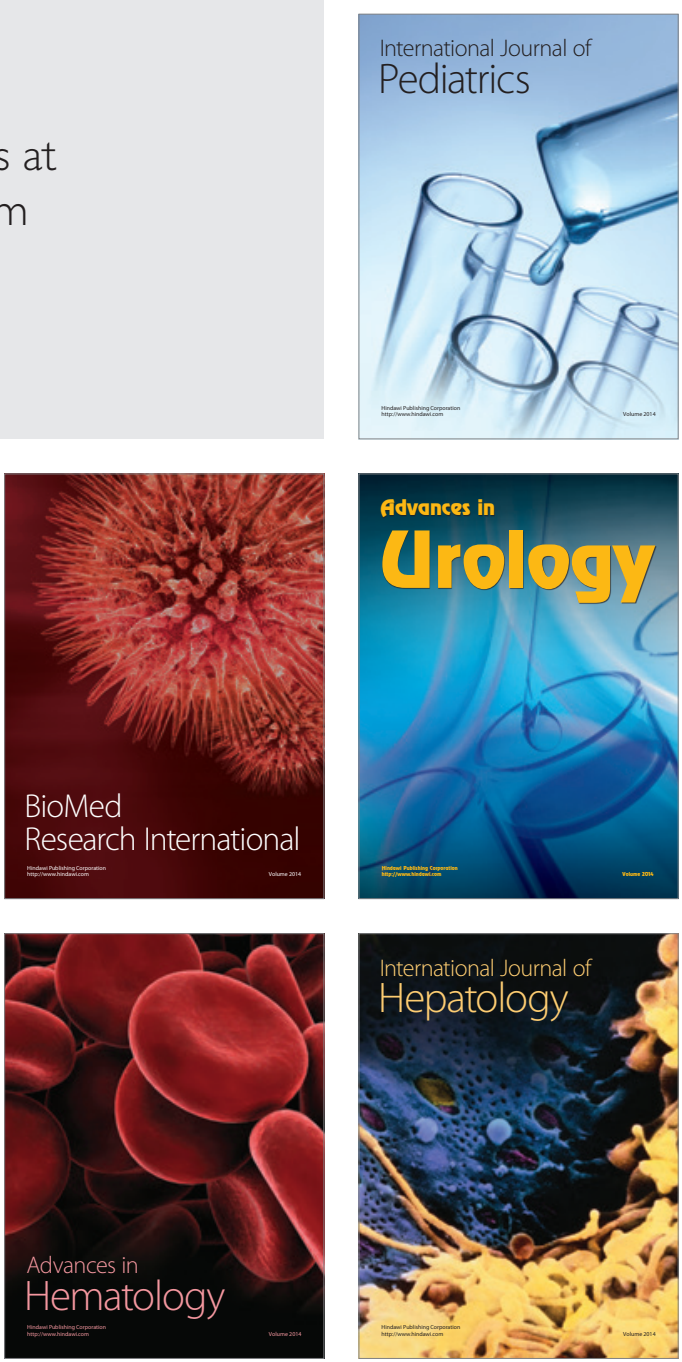